\title{
Reliable Bounding Zones and Inconsistency Measures for GPS Positioning Using Geometrical Constraints*
}

\author{
Hani Dbouk ${ }^{a}$ and Steffen Schön ${ }^{a}$
}

\begin{abstract}
Reliable confidence domains for positioning with Global Navigation Satellite System (GNSS) and inconsistency measures for the observations are of great importance for any navigation system, especially for safety critical applications. In this work, deterministic error bounds are introduced in form of intervals to assess remaining observation errors. The intervals can be determined based on expert knowledge or - as in our case - based on a sensitivity analysis of the measurement correction process. Using convex optimization, bounding zones are computed for GPS positioning, which satisfy the geometrical constraints imposed by the observation intervals. The bounding zone is a convex polytope. When exploiting only the navigation geometry, a confidence domain is computed in form of a zonotope. We show that the relative volume between the polytope and the zonotope can be considered as an inconsistency measure. A small polytope volume indicates bad consistency of the observations. In extreme cases, empty sets are obtained which indicates large outliers. We explain how shape and volume of the polytopes are related to the positioning geometry. Furthermore, we propose a new concept of Minimum Detectable Biases.

Using the example of the Klobuchar ionospheric model and Saastamoinen tropospheric model, we show how observation intervals can be determined via sensitivity analysis of these correction models for a real measurement campaign. Taking GPS code data from simulations and real experiments, a comparison analysis between the proposed deterministic bounding method and the classical least-squares adjustment has been conducted in terms of accuracy and reliability. It shows that the computed polytopes always enclose the reference trajectory. In case of large outliers, large position deviations persist in the least-squares solution while the polytope algorithm yields empty sets and thus successfully detects the cases with outliers.
\end{abstract}

Keywords: GPS, polytope, zonotope, integrity, reliability, intervals

\footnotetext{
*This work was supported by the German Research Foundation (DFG) as a part of the Research Training Group i.c.sens [GRK2159].

${ }^{a}$ Leibniz Universität Hannover, Institut für Erdmessung, Schneiderberg 50, 30167 Hannover, Germany, E-mail: \{dbouk, schoen\}@ife.uni-hannover.de
} 


\section{Introduction}

Nowadays, GNSS is applied for a variety of highly demanding tasks, like e.g., precision landing approaches. Thus, the quality and trust that we put into the GPS navigation solution must be extremely high: Integrity measures this performance, i.e. the ability of the navigation system to timely warn the user when error thresholds so-called alert limits are transgressed [23].

Integrity risk evaluation involves, both assessing the fault detection and exclusion capability and quantifying the impact of undetected faults on position estimation errors. Currently, there is a big interest in receiver autonomous integrity measures (RAIM) algorithms especially for autonomous driving applications [3, 27].

This purely statistical determination of integrity and associated error bounds will not always be adequate. In the past years, new integrity approaches have been proposed based on interval mathematics, e.g. Set Inversion Via Interval Analysis is used to compute the three-dimensional bounding zone in real time $[13,12]$. This approach differs from the usual Gaussian error model, since the uncertainty of the satellite positions and the pseudo-range measurements is assessed by intervals which bound the true values of the satellite positions and the pseudo-range measurements with a particular confidence. Subsequently, the user position will be guaranteed to be in a bounding box, with edges parallel to the axes of the coordinate system which is a direct solution of the non-linear navigation equation, [6], [11] and [8]. The drawbacks of this approach are: (i) the obtained bounding box overestimates the uncertainty region, (ii) the size and shape of the bounding box depends on the orientation of the coordinate system and thus the uncertainty may be not well presented, (iii) a concept of minimum detectable biases (MDB) is not foreseen.

In this work, we propose a different deterministic bounding method which takes benefits from the geometry of the navigation problem. The bounds on the observation equations represent constraints for the parameters that have to be satisfied. As a result, a convex polytope is obtained which can overcome some of the aforementioned shortcomings.

The remainder of the paper is structured as follows: The first section is about the methodology which contains some basics on GNSS positioning, polytopes and zonotopes, the determination of observation interval radii from sensitivity analysis of the GPS correction models, and the transformation of the navigation problem into a convex polytope problem by applying the interval bounds in terms of geometrical constraints. The second section includes a simulation study to better understand the properties of our method. Then, we derive the MDB and we explain the effect of biases on the bounding zone as well. Subsequently, the inconsistency measure is derived and studied in terms of geometry, error bounds, and observation noise also using real and simulated GPS code measurements. In the third section, real GPS code data from kinematic test drives will be analyzed, [19]. We compute the interval radii for the code observations based on a sensitivity analysis of two prominent correction models. We show exemplary trajectories for which the method based on geometric constraints is compared to the classical least-squares estimator and subsequent integrity measures. 


\section{Methodology}

\subsection{Basics on GNSS Positioning}

The GNSS position concept is based on pseudo-range measurements $P R$ between the known satellite positions $\mathbf{x}^{j}=\left(x_{j}, y_{j}, z_{j}\right)^{T}$ and the unknown user position $\mathbf{x}_{A}=\left(x_{A}, y_{A}, z_{A}\right)^{T}$. Since the receiver clock is not synchronized with the GNSS time scale, the receiver clock offset $\delta_{A}(t)$ needs to be estimated epoch by epoch as a fourth parameter and only pseudo-ranges instead of ranges can be measured [17]. In addition, the signals are delayed. The main sources of errors come from satellites ephemerides, propagation delays through the atmosphere, receiver noise, multipath and interfering signals [26]. Moreover, satellite anomalies and outliers occur and should be detected and excluded from the computation in order to have a reliable positioning. The code pseudo-ranges equation (1) for satellite $j$ and station $A$ reads

$$
\begin{aligned}
P R_{A}^{j}(t) & =\sqrt{\left(x_{j}\left(t-\tau_{A}^{j}\right)-x_{A}(t)\right)^{2}+\left(y_{j}\left(t-\tau_{A}^{j}\right)-y_{A}(t)\right)^{2}+\left(z_{j}\left(t-\tau_{A}^{j}\right)-z_{A}(t)\right)^{2}} \\
& +c\left(\delta_{A}(t)-\delta_{j}\left(t-\tau_{A}^{j}\right)\right)+I_{A}^{j}(t)+T_{A}^{j}(t)+M_{A}^{j}(t)+w(t),
\end{aligned}
$$

where, $t$ is the epoch of reception, $t-\tau_{A}^{j}$ the transmission time, $\tau_{A}^{j}$ the signal travel time, $x, y$ and $z$ are the coordinates in an Earth centered Earth fixed (ECEF) coordinate system, $c$ denotes the speed of light, $\delta_{j}$ satellite clock offset, $I_{A}^{j}(t)$ and $T_{A}^{j}(t)$ denote the ionospheric and tropospheric delays respectively, $M_{A}^{j}(t)$ is the multipath error and $w$ contains the remaining errors. At least four satellites in view are needed in order to estimate the receiver position and clock offset. After linearization via Taylor expansion at some initial values $\mathbf{x}_{0}$, we get the over-determined system of linearized observation equations at every epoch:

$$
\Delta l=\mathbf{A} \Delta \mathbf{x}
$$

where $\Delta \boldsymbol{l}$ denotes the $m \times 1$ vector of observed-minus-computed values (OMC), $\mathbf{A}$ the $m \times 4$ design matrix and $\Delta \mathbf{x}$ the $4 \times 1$ vector of corrections to be estimated and added to $\mathbf{x}_{0}$. In general, the weighted least-squares principle is applied to obtain best estimates (3), where the observations are weighted depending on the satellite elevation angle $E l\left(\mathbf{P}=\operatorname{diag}\left(\sin ^{2}(E l)\right)\right)$, thus taking into account potentially larger deviations at low elevation angles

$$
\Delta \hat{\mathbf{x}}=\left(\mathbf{A}^{\mathbf{T}} \mathbf{P} \mathbf{A}\right)^{-1} \mathbf{A}^{\mathbf{T}} \mathbf{P} \Delta l
$$

\subsection{Basics on Polytopes and Zonotopes}

Following [28], a polyhedron is defined as the solution set of a finite number of linear equalities and inequalities

$$
P=\left\{\mathbf{x} \mid \mathbf{n}_{\mathbf{i}}^{\mathbf{T}} \mathbf{x} \leq u_{i}, i=1, \ldots, m, \quad \mathbf{c}_{\mathbf{i}}^{\mathbf{T}} \mathbf{x}=d_{i}, i=1, \ldots, p\right\} .
$$


The polyhedron can also be defined as the convex hull of a finite number of points which represent its vertices

$$
P=\operatorname{conv}\left(\mathbf{v}_{\mathbf{0}}, \mathbf{v}_{\mathbf{1}}, \ldots, \mathbf{v}_{\mathbf{m}}\right)=\left\{\theta_{0} \mathbf{v}_{\mathbf{0}}, \theta_{1} \mathbf{v}_{\mathbf{1}}, \ldots \theta_{m} \mathbf{v}_{\mathbf{m}} \mid \boldsymbol{\theta} \geq \mathbf{0}, \mathbf{1}^{\mathbf{T}} \boldsymbol{\theta}=\mathbf{1}\right\} .
$$

A polyhedron is the intersection of a finite number of halfspaces and hyperplanes. In this paper, we will follow the convention of calling a bounded polyhedron a polytope. Zonotopes or parallelotopes are special polytopes with specific geometrical properties, e.g. pairs of edges have the same length and are parallel. A good reference for zonotopes and its uses in uncertainty estimation are [28] and [20].

\subsection{Determination of the Bounding Zones}

The transformation of the navigation problem to a convex polytope is done by applying the error bounds $\Delta$ on OMC $(\Delta l)$ in both directions, so equation (2) reads as follows:

$$
\Delta \boldsymbol{l}-\Delta \leq \mathbf{A} \Delta \mathrm{x} \leq \Delta \boldsymbol{l}+\Delta,
$$

The determination of the interval radii as error bounds will be explained in the next session. Rearranging equation (6), we get a system of inequalities:

$$
\left\{\begin{array}{l}
\mathbf{A} \Delta \mathbf{x} \leq \Delta l+\Delta \\
-\mathbf{A} \Delta \mathbf{x} \leq-\Delta l+\Delta
\end{array}\right.
$$

which can be written as follows:

$$
\mathbf{B} \Delta \mathbf{x} \leq \mathbf{b}
$$

where $\mathbf{B}=\left[\begin{array}{c}\mathbf{A} \\ -\mathbf{A}\end{array}\right]$ and $\mathbf{b}=\left[\begin{array}{c}\Delta \boldsymbol{l}+\Delta \\ -\Delta \boldsymbol{l}+\Delta\end{array}\right]$

This system of inequality constraints defining a convex polytope as an intersection of a finite number of halfspaces is referred to as a H-polytope. Each inequality constraint is a halfspace. To get the vertices of the polytopal bounding zone in the coordinate system, the H-polytope has to be transformed into a V-polytope (convex hull of the polytope vertices). This transformation is the so-called vertex enumeration problem, and there exist different algorithms to solve it. A Primal Dual Polytope (PDP) algorithm is used to transform the H-polytope into a VPolytope. In this paper, we use the Multi-Parametric MatLab Toolbox [9]. Figure 1 shows a $2 \mathrm{D}$ example of the transformation from H-polytope into V-polytope, where each row of the matrix $\mathbf{B}$ represents a normal vector of the hyperplane of the H-polytope. In this example, the measurements are error free i.e., $\Delta \boldsymbol{l}=0$ and that is why the polytope is regular with special properties (zonotope) [28].

The volume of the polytope will be used later on to derive the inconsistency measures of the positioning problem. There is no analytical form to compute the volume of non-regular polytopes. However, different methods and algorithms exist to derive the polytope volume numerically, as discussed in [4], [16], and [5]. Contrary, the zonotope volume can be computed analytically [28]. In this paper, the volumes of the polytopes and zonotopes will be computed by the same MatLab built-in algorithm in order to avoid inconsistency in the computation. 


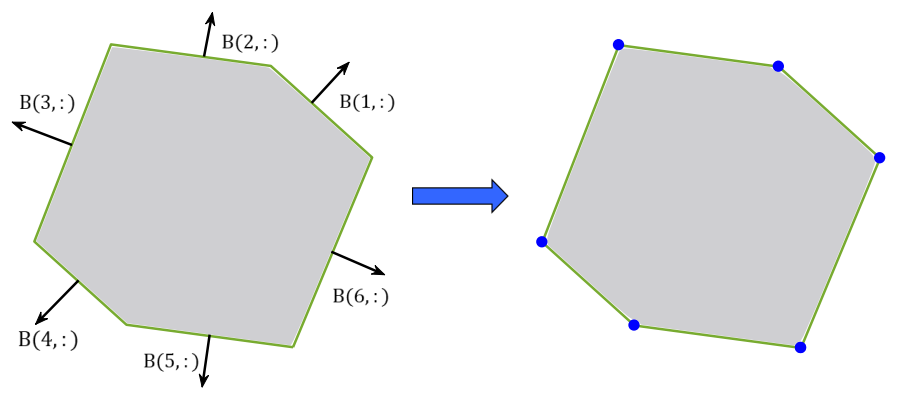

Figure 1: H-Polytope (left): intersection of halfspaces (grey area) determined by 6 different hyperplanes which are the rows of a matrix $\mathbf{B}$ and its transformation into a V-polytope (right) blue dots.

\section{$2.4 \quad$ Determining Observation Intervals}

In our approach, error bounds in form of intervals with lower and upper bounds are applied to the $\mathrm{OMC}$ values $\boldsymbol{\Delta} \boldsymbol{l}$ to assess the remaining observation uncertainty. A sensitivity analysis w.r.t influence parameters is performed on the observation equation (1) to derive observation intervals [21]. Each correction model in equation (1) is checked w.r.t influence parameters whose values are known at some time step with some uncertainty. Usually, there are both systematic and random effects.

The dependencies of the total differential $d P R_{A}^{j}$ of equation (1) w.r.t. the influence parameters are defined as follows.

$$
d P R_{A}^{j}=\frac{\partial \rho_{A}^{j}}{\partial \mathbf{s}} \mathbf{d} \mathbf{s}+\frac{\partial I_{A}^{j}}{\partial \mathbf{s}} \mathbf{d} \mathbf{s}+\frac{\partial T_{A}^{j}}{\partial \mathbf{s}} \mathbf{d} \mathbf{s}+\ldots=\mathbf{F} \mathbf{d} \mathbf{s}
$$

where $\rho_{A}^{j}$ denotes the Euclidean distance, the $\left(n_{\text {infl }} \times 1\right)$ vector $\mathbf{s}$ is the set of influence parameters and $\mathbf{d s}$ is their uncertainty. The final interval radius $\Delta_{A}^{j}$ is defined :

$$
\Delta_{A}^{j}=\left|\frac{\partial P R_{A}^{j}}{\partial \mathbf{s}}\right| \mathbf{d} \mathbf{s}=|\mathbf{F}| \mathbf{d} \mathbf{s},
$$

where $\mathbf{F}$ is a matrix containing the partial derivatives, the symbol $|\cdot|$ is the absolute value applied to each matrix element. We assume, that the error bounds are symmetric and centered at the actual value of the corrected observations $\Delta l_{A}^{j}$, so the interval observation reads as follows:

$$
\left[\Delta l_{A}^{j}\right]=\left[\Delta l_{A}^{j}-\Delta_{A}^{j}, \Delta l_{A}^{j}+\Delta_{A}^{j}\right]
$$

\section{Interpretation of Bounding Zones and related Inconsistency Measures}

In the following, different case studies will be analyzed for better understanding the shape and interpretation of the resulting polytopes. A simulation study of a moving 
robot in a flat world will be presented, taking into account special positioning geometries, measurement noise and biases. We assume that the transmitters are fixed in each scenario in a known position and can communicate with the robot via electromagnetic waves as GNSS satellites and receiver. We assume further that the transmitters and the robot are synchronized, so that the robot position is the only state to be estimated.

\subsection{Noise-free and Bias-free Measurements Yield Zonotope as Bounding Zone}

Figure 2 shows three simulated scenarios with different transmitter geometries (transmitters are fixed in each case) thus different angles of intersection between the lines-of-sight (LOSs). The measurements are considered to be free of random errors and fit the model $\Delta \boldsymbol{l}=0$. So, we can have a better idea of the impact of the navigation geometry on the bounding zone. In all cases, the observation error bound is selected arbitrary and set equal to 4 meters.

When changing the navigation geometry, i.e. going from a good, homogeneous distribution of the transmitters (case a) to a worse, more clustered one (case c), the area of the polytope increases, reflecting this situation. In this example, the area of the polytope (i.e. zonotope) is $56 \mathrm{~m}^{2}, 82.3 \mathrm{~m}^{2}$, and $278.1 \mathrm{~m}^{2}$ for the cases a, b, and c, respectively. This reflects the impact of the positioning geometry also presented in the Geometrical Dilution of Precision (GDOP) values $\left(G D O P=\sqrt{\operatorname{trace}\left(\mathbf{A}^{\mathbf{T}} \mathbf{A}\right)^{-1}}\right)$ used in navigation to characterize the strength of the navigation geometry [15]. Here, the values are GDOP $=1.2,1.7$ and 12.4 for case $\mathrm{a}, \mathrm{b}$ and $\mathrm{c}$, respectively.
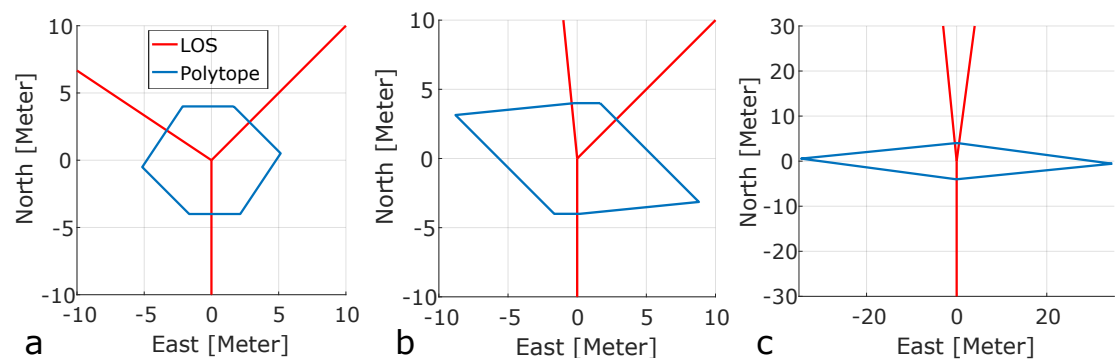

Figure 2: Resulting bounding zones (blue) for the error-free case. 2D simulated scenarios with error bounds equal to $4 \mathrm{~m}$ and 3 transmitters with different geometry (LOS). a) excellent geometry $\mathrm{GDOP}=1.2$, polytope area equals $56 \mathrm{~m}^{2}, \mathrm{~b}$, intermediate geometry $\mathrm{GDOP}=1.7$, polytope area equals $82.3 \mathrm{~m}^{2}$, c, bad geometry $\mathrm{GDOP}=12.4$, polytope area equals $278.1 \mathrm{~m}^{2}$

Since neither observation noise nor biases are applied here, we consider the zonotope as the nominal uncertainty associated with a specific geometry and value for $\Delta$. Furthermore, the shape of the zonotope indicates directions in which the point uncertainty is minimum, i.e. in LOS direction. The number of edges and vertices of a $2 \mathrm{D}$ zonotope equals twice the number of measurements. 


\subsection{Impact of White Observation Noise on the Bounding Zone}

When adding noise to the observations, the zonotope is deformed into a polytope, cf. Figure 3.a. The degree of deformation depends on the ratio between the applied error bounds $\Delta$ and the standard deviation of the observations $\sigma$. In order to study this impact, this ratio is varied while keeping the identical geometry.

Figure 3.b, shows the resulting bounding zones of the robot in an example with six well distributed transmitters. The colored polytopes are obtained from the same set of transmitters but from measurements suffering from additive white noise (AWN, $\sigma=2 \mathrm{~m}$ ). Furthermore, the interval $\Delta$ is increased. As a reference, the polytopes without AWN (i.e. zonotopes) are shown in black. The big one is obtained when applying $\Delta=16 \mathrm{~m}$ while the small one for $\Delta=4 \mathrm{~m}$. The two black zonotopes are similar and both have 12 edges.

Starting with the large zonotope, the situation changes as follows. As the size of $\Delta$ decreases, the variations due to AWN start predominating. Subsequently, the number of edges decreases since each constraint cuts the polytope from inside, cf. dashed-lined edges. The color of dashed-lined edges indicates at which magnitude of the error bound $\Delta$ that specific edge is lost. For instance, edge 1 is lost from the error free case when we add an AWN to the measurements with $\Delta=16 \mathrm{~m}$, while edge 2 is lost when $\Delta=4 \mathrm{~m}$, (magenta polytope). Overall, the black zonotope, blue, red, green, and magenta polytopes have $12,11,10,10$, and 8 edges, respectively. As the ratio $\frac{\Delta}{\sigma}$ decreases, the number of edges is reduced and the area of the polytope decreases till it becomes empty. Thus, the area or volume of the polytope $\operatorname{Vol}_{P}$ can be used to indicate the inconsistency of the observations, here generated by AWN. Next we treat the impact of biases.

\subsection{Impact of Observation Biases on the Bounding Zone}

Let's go back to the 2D example represented in Figure 2, and add one new transmitter, so one more measurement. Assuming noise-free measurements but a biased new measurement, the impact of an increase in magnitude of the bias is studied depending on the observation geometry. Figure 4.a depicts the zonotopes for the error-free cases for three (blue) and four (green) transmitters (Tx).

Figure 4.b and c show the polytope solution when we introduce biases to the $4^{\text {th }}$ observation with different magnitudes related to $\Delta$. The bias acts as a halfspace cut in the LOS direction (depicted in red). As the bias increases the resulting polytope moves in the direction of the bias and shrinks down till it becomes empty at a bias $\delta=\Delta+w$, where $w$ is the half width of the blue zonotope, [28]. When the bias is just a bit smaller than $\Delta+w$, the resulting polytope is very small, see Figure 4.c, black triangle at the left. This underlines again the interpretation of the polytope being an inconsistency measure.

In case $\mathrm{c}$ from Figure 2 , we also take the $4^{\text {th }}$ transmitter in the direction of maximum uncertainty. In this case, $w$ is much bigger than the one from case a. In fact, the polytope is still non-empty for a very large bias (see black trapezoid in Figure 4.e), 

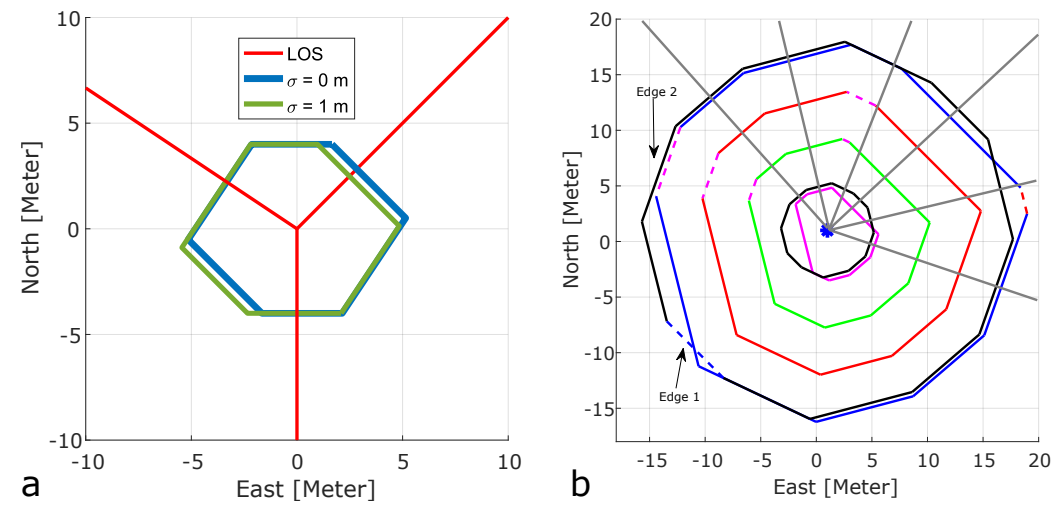

Figure 3: Comparison of zonotope and polytope solutions: a) Impact of noise on a zonotope. b) Polytope solutions with different values for the error bounds of $4 \mathrm{~m}, 8$ $\mathrm{m}, 12 \mathrm{~m}$, and $16 \mathrm{~m}$, while keeping the same noise level. The black regular polytope (zonotope) is the solution obtained from measurements free of random errors (big zonotope is obtained with error bound $\Delta=16 \mathrm{~m}$, the small one is of $\Delta=4 \mathrm{~m}$ ), while the colored non-regular polytopes result from measurements affected with AWN $(\sigma=2 \mathrm{~m})$ and varying $\Delta$. The grey lines indicate the line-of-sight directions.

where a bias of $\delta=-7 \cdot \Delta=-28 \mathrm{~m}$ was introduced. Thus, this underlines the strong impact of the observation geometry.

Now, we can define a minimum detectable bias $\left(M D B_{Z_{i}}\right)$ being an error in observation $i$ so that the resulting polytope is an empty set. From geometric consideration, the $M D B_{Z_{i}}$ equals the sum of the error bound $\Delta_{i}$ and the half width $w_{i}$ of the zonotope $Z_{i}$ in the LOS direction of the corresponding observation $i$, where the zonotope is obtained from all observations except the $i^{t h}$ one. The half width of the zonotope is the half distance between the extreme vertices of the zonotope in the LOS direction of the related observation, cf. Figure 5 for the geometric relations.

The MDB is maximum if the LOS of the new measurement is in the direction of maximum uncertainty of the zonotope. The big advantage is that the MDB can be computed without real measurements as a design and planning quantity. It indicates the strength of the navigation geometry to identify outliers.

$$
\left\{\begin{array}{l}
M D B_{Z_{i}}=w_{z_{i}}+\Delta_{i} \\
\Delta_{i} \leq M D B_{P_{i}} \leq 2 w_{p_{i}}+\Delta_{i}
\end{array}\right.
$$

Real observations however will be noisy. Thus, the resulting bounding zone is a polytope. If the actual noise contribution in each measurement is smaller than the corresponding error bound, then the true position is still contained inside the polytope but its location is unknown. Thus, only a range of MDB can be given by $M D B_{P_{i}}$ (Equation 12) where $w_{p_{i}}$ is the half width of the polytope obtained with all observations except the $i^{t h}$ one. If the noise contribution is small w.r.t the error 
bounds, the polytope solution tends towards the zonotope solution.

In the context of the least-squares adjustment, the MDB is derived from hypothesis testing of individual outliers in observations:

$$
M D B_{T S_{i}}=\frac{\sigma_{0}}{p_{i}} \cdot \sqrt{\frac{\lambda_{i}}{q_{v_{i} v_{i}}}}
$$

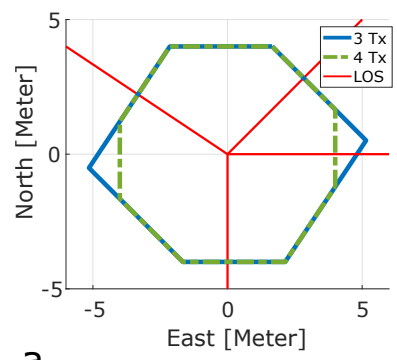

a
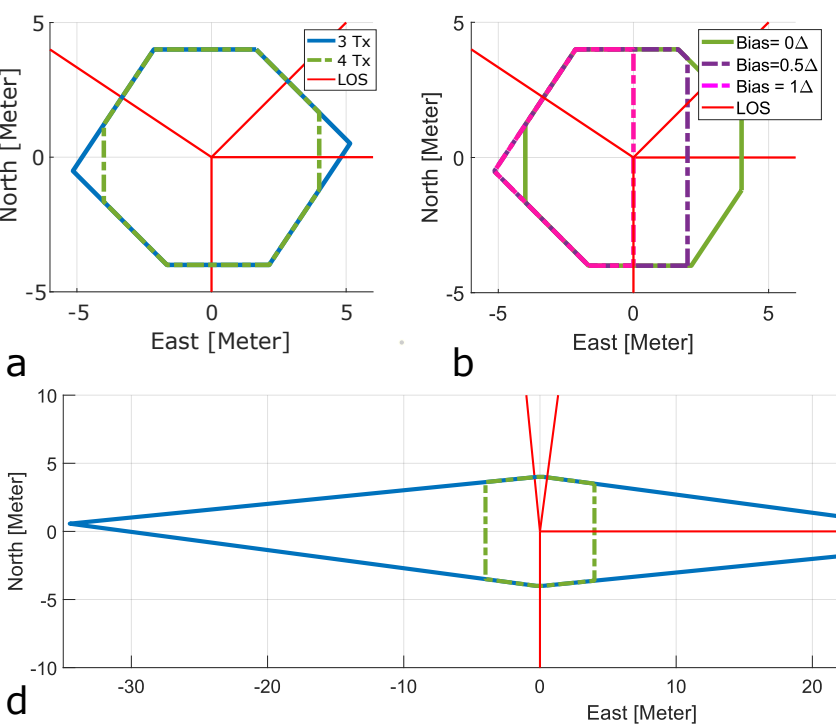

b

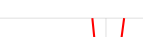

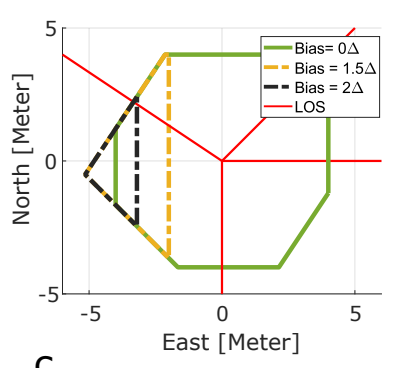

C

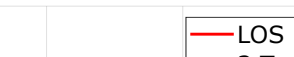

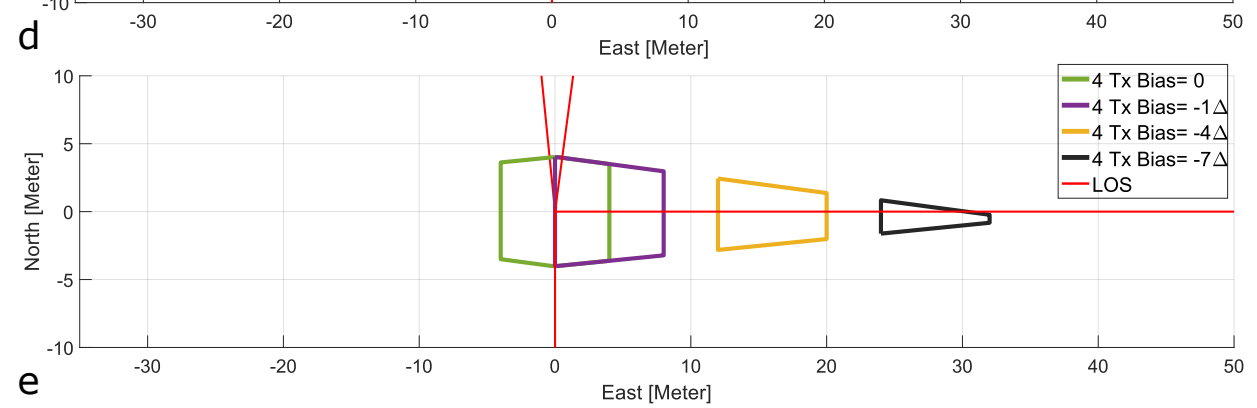

Figure 4: Introducing a range of biases to the $4^{\text {th }}$ measurement of case a and c from Figure 2. Subfigure a) regular polytope (zonotope) before (blue) and after (green) introducing the $4^{\text {th }}$ observation. b) Biases $\delta=0.5 \Delta$ and $\delta=1 \Delta$ introduced to the $4^{\text {th }}$ observation of case a. c) Biases $\delta=1.5 \Delta$ and $\delta=2 \Delta$ introduced to the $4^{t h}$ observation of case a. d) Introducing a $4^{\text {th }}$ observation in case c of Figure 2. e) Range of biases $\left(\delta=1 \Delta, 4 \Delta\right.$, and $7 \Delta$ ) added to the $4^{\text {th }}$ observation. Zonotopes computed with only 3 observations are shown in blue and LOSs of the observations are indicated in red. 

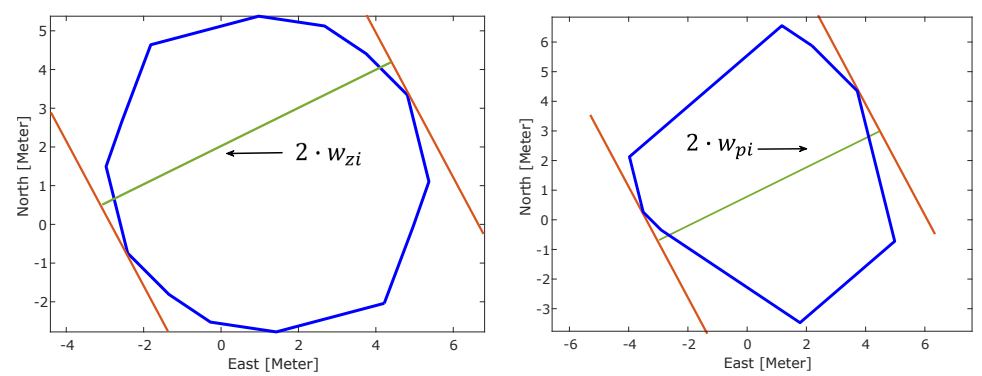

Figure 5: Geometry of the width of the zonotope (a) and polytope (b)

where $\sigma_{0}$ is the prior standard deviation of the observation, $p_{i}$ is the weight of the $i^{t h}$ observation which depends on the elevation angle, $\lambda_{i}$ is the non-centrality parameter, $q_{v_{i} v_{i}}$ is the estimated variance of the $i^{\text {th }}$ observation's residual, $\mathbf{v}$ is the vector of residuals and $\mathbf{P}$ is the weight matrix, [24] and [1]. Assuming normal distributed observation errors, the non-centrality parameter depends on the chosen probability of false alarm (the significance level) and the detection power (test quality) that separates the hypothesis $H_{0}$ : case of no fault from the faulty case (alternative hypothesis $H_{1}$ ), [25]. In general, an overall model test or global test is preceding the individual outlier test:

$$
T_{g l}=\frac{\mathbf{v}^{\mathbf{T}} \mathbf{P} \mathbf{v}}{m-4}:\left\{\begin{array}{l}
H_{0} \sim \chi_{m-4}^{2} \\
H_{1} \sim \chi_{m-4, \lambda_{0}}^{2}
\end{array}\right.
$$

The global test statistics $T_{g l}$ follows a central $\chi^{2}$ distribution of $f=m-4$ degrees of freedom in the fault-free case $H_{0}$ and a non central $\chi^{2}$ distribution in the faulty case. In order to ensure equal sensitivity of both tests, the probabilities of error and the non-centrality parameters $\lambda_{0}$ and $\lambda_{i}$ must be adjusted, cf. [24] and [1].

\subsection{Proposal for an extended Inconsistency Measure}

As we have seen in the previous section, there are some biases that could not be detected just by empty sets. These non-detectable biases (NDBs) have values between $\Delta$ and $\Delta+2 w$. This type of biases is dangerous because we still have a non-empty polytope solution and the polytope may not contain the true solution. This is especially true for a bad remaining geometry or less redundancy for a specific observation, e.g. compare case $\delta=-4 \cdot \Delta$ in figure 4 e).

To detect such biases we propose a new inconsistency measure $V_{r}$ based on the relative volume between the volume of the actual polytope solution $\operatorname{Vol}_{p}$ and the error and bias-free solution (reference or nominal behavior), i.e. the zonotope $V_{o l}$ :

$$
V_{r}=\frac{V o l_{z}-V o l_{p}}{V o l_{z}} .
$$

We perform a Monte Carlo simulation to understand the behavior of the inconsistency measure in terms of geometry, noise, error bounds and biases. Three different 
real satellite geometries (scenarios) are selected with different number of satellites in view and geometrical dilution of precision GDOP (Table 1). We simulate GPS code measurements with white noise $(\sigma=1 \mathrm{~m})$. In each of the 1000 simulation runs (epochs), a different random vector is generated, however the sequence of random vectors are identical for all three scenarios. Finally, a ramp bias is introduced starting from epoch $100(\delta=0 \mathrm{~m})$ and ending at epoch $500(\delta=32 \mathrm{~m})$.

Table 1: GDOP and number of satellites for each scenario in the Monte Carlo simulation.

\begin{tabular}{c|ccc} 
Scenario & 1 & 2 & 3 \\
\hline Number of Satellites & 10 & 6 & 5 \\
GDOP & 2.0 & 3.4 & 11.3
\end{tabular}

Figure 6.a shows the impact of different satellite configurations when the error bounds $\Delta$ are fixed to $4 \mathrm{~m}$ and one satellite (PRN10) was biased. For Scenario 1 (good geometry, blue): the inconsistency measure $V_{r}$ increases as expected, when the bias increases. If the bias transgresses a certain amount (here $9 \mathrm{~m}$ ), the polytope is empty, i.e. the bias is successfully detected. During the other epochs without bias, $V_{r}$ shows a certain scatter, which is related to the selected random vectors and which can be used to define a nominal behavior. Scenario 2 with intermediate geometry (orange) shows an increase in the inconsistency at the beginning of the ramp bias. $V_{r}$ can successfully detect biases larger than $13 \mathrm{~m}$. A totally different behavior is obtained for Scenario 3. For small biases, $V_{r}$ increases already strongly and then a large scatter but no empty sets are observed. The scatter in the inconsistency measures in the non-biased regions is higher for the good geometry and lower for bad geometry. This is due to the fact that for a bad geometry zonotopes and polytopes are larger so that the observation noise has a smaller impact on the polytope volume.

In Figure 6.b the impact of a varying magnitude of the error bounds $\Delta$ is shown for Scenario (1) and the biased satellite (PRN 10). As expected, the inconsistency measure $V_{r}$ behaves in a different way with and without biases. As the error bound increases the mean value and the scatter of $V_{r}$ decreases since the impact of the measurement noise on the polytope volume decreases, too. During epochs with bias, the slope of $V_{r}$ decreases. Thus, the measure becomes more and more insensitive against inconsistencies and outliers can hardly be detected, i.e. the number of empty set solutions is significantly reduced.

Figures 6.c and 6.d display the impact of different biased satellites where the geometry and the error bounds are kept fixed. Figure 6.c depicts a good geometry situation (Scenario 1; GDOP $=2.0$ ) and reveals the same effect for different biased satellites, while Figure 6.d depicts the bad geometry case (Scenario 3; GDOP = 11.3). Consequently, the zonotope and polytope are large and elongated, since the LOS directions are not well distributed and rather clustered in one direction. This implies a different behavior of the inconsistency measure for different biased satel- 
lites, which depends on the line-of-sight direction of the biased satellites and the geometry of the other satellites in view. Moreover, the detection was not possible here for the large error bound of $10 \mathrm{~m}$, see also [7] for a discussion.

In order to improve the sensitivity of the inconsistency measure, a first approach is to introduce an upper threshold for $V_{r}$ to reject biased epochs. To this end, a nominal behavior is needed to compare the current situation against. We propose to run a MC simulation taking the real observation geometry into account as well as the adequately defined error bound $\Delta$ and standard deviation $\sigma$ thus obtaining a nominal behavior. Subsequently, a histogram of $V_{r}$ are determined and thresholds can be set. As motivated above, small values of $V_{r}$ can be generated by outliers and/or bad geometry. Thus, it is advised to exclude low values of $V_{r}$, too.

$$
\text { exclude epochs if } V_{r}<V_{r, l} \text { or } V_{r}>V_{r, u}
$$
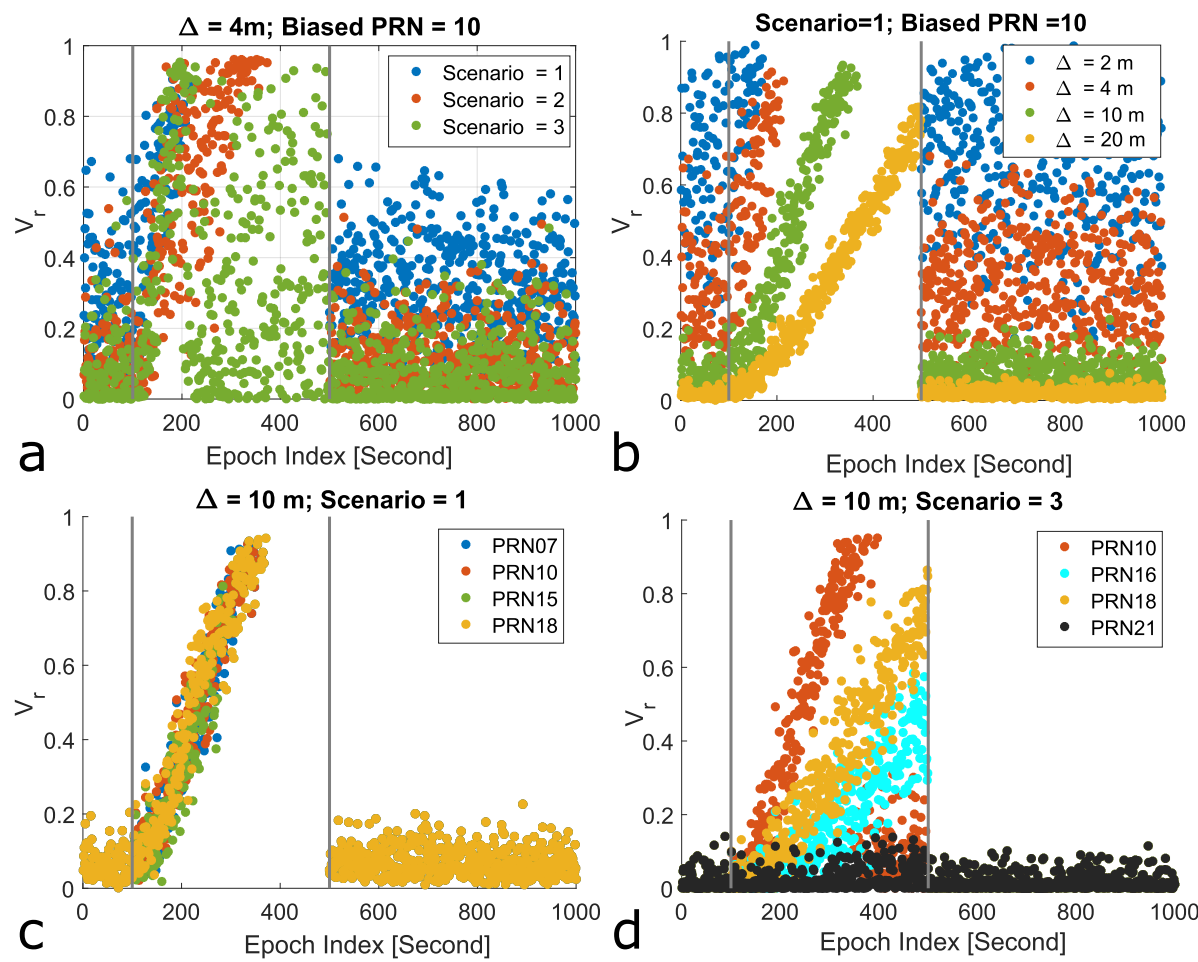

Figure 6: Variation of the inconsistency measure for real GPS configurations with simulated measurements with AWN (1m) and a ramp bias for one satellite between epoch 100 and 500. a: Impact of satellite geometry for 3 different scenarios, b: Impact of varying magnitude of error bounds $\Delta$ in case of good satellite geometry, c: Impact of different biased satellites for a large error bound of $\Delta=10 \mathrm{~m}$ and a good geometry, , d: same as c but for Scenario 3 (bad geometry). 


\section{Real Data Analysis}

Intensive test drives have been done in the scope of DFG funded research training group I.C.SENS, [19]. In this study, we will present one of those car test drives. The chosen test drive was recorded in a GNSS denied urban area (Figure 8.b) using a Novatel Span system consisting of a dual frequency GNSS receiver [22] equipped with 2 antennas and an iMAR FSAS IMU [10]. The ground truth is provided by the post processing software TerraPos [2], where the very precise carrier phase observations of the 2 GNSS antennas were tightly coupled with the IMU measurements.

\subsection{Computation of Observation Intervals from Sensitivity Analysis}

Exemplary, the observation intervals are computed by a sensitivity analysis for the relevant Klobuchar [14] ionospheric model and the Saastamoinen [18] tropospheric model in order to derive physically meaningful interval bounds. The uncertainty values of the influence parameters can be found in [7,21]. The remaining errors of the GPS code observation are enclosed by an error bound equal to the typical observation error of $1 \%$ of the chip or wavelength, i.e. $\sim 3 \mathrm{~m}$. Subsequently, the elevation dependent interval bounds are determined, cf. Figure 7.

The right hand part shows the skyplot, i.e. the satellite distribution during the measurement campaign. The left hand part depicts the interval radii, color coded for each satellite. For high elevating satellites the interval radii are smaller (about $12 \mathrm{~m}$ ) while data from low elevation satellites is more uncertain which is reflected by larger interval radii of up to $17 \mathrm{~m}$. Interruption in the time series indicates that the corresponding satellite was not measured at that epoch due to heavy obstructions in this urban areas.
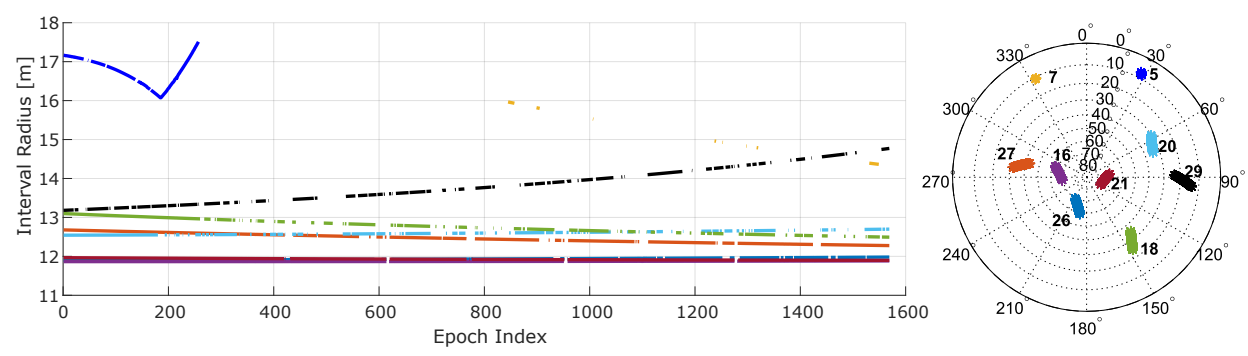

Figure 7: Interval radius of each observation derived from sensitivity analysis and the skyplot of the test drive.

In order to link the proposed approach to the classical least-squares adjustment the following statements can be made: (i) Assuming normal distribution and a typical standard deviation of $\sigma=3 \mathrm{~m}$ for a GPS observation at zenith direction, a confidence interval of radius $12 \mathrm{~m}$ would contain more than $99.99 \%$ of the observations. 
(ii) The ratio $\frac{\Delta}{\sigma}$ varies due to the elevation depending interval radii and observation weights from 4 (zenith) to 1 at low elevation (e.g. 10 degrees).

\subsection{Positioning solutions}

Three positioning solutions are computed from the GPS code observations: (i) a classical least-squares solution (Eq. 3) with global test (Eq. 14) and significance level of $0.2 \%$, (ii) the proposed polytope solution (Eq. 8 ) based on the before determined observation intervals without threshold and (iii) with thresholds on $V_{r}$ here: $0.04<$ $V_{r}<0.4$. All solutions are compared to the reference trajectory.

Figure 8.a shows the obtained results. The reference solution is depicted in black, the epoch-wise single point positions (SPP) obtained from the classical least-squares solution in blue and our polytope solution in green. In addition, as potential point measure, the barycentres of the polytopes are indicated in orange. Both methods provide noisy solutions w.r.t. the reference, since the rather noisy GPS code measurements have been used. All the bounding polytopes enclose the reference solution. Figure 9 shows the cumulative frequency of the 3D positioning error for the three cases: least-squares adjustment (LSA, blue), polytope-based point position without thresholds (PDP, red) and polytope-based point position with thresholds $\left(P D P_{T}\right.$, dashed red). Both solutions based on polytopes outperform the LSA solution, e.g. a 3D position error less than $10 \mathrm{~m}$ is obtained in $38 \%, 42 \%$ and $60 \%$ of the epochs of $L S A, P D P$ and $P D P_{T}$, respectively. For comparison the LSA solution is indicated as dashed blue line when only the epochs of common to the $P D P_{T}$ solution are considered. Here in 584 of the epochs the $3 \mathrm{D}$ position error is smaller than $10 \mathrm{~m}$. The root mean square errors are $14 \mathrm{~m}, 12.93 \mathrm{~m}, 9.98$ and 9.3 $\mathrm{m}$ for $L S A, P D P, L S A_{T}$ and $P D P_{T}$, respectively. It is worth mentioning that the total number of epochs was 1606 where 74 epochs have less than 4 satellites in view so the position could not be computed. From the remaining 1532 epochs, the global test fails at 223 epochs, while we get 94 empty polytopes and 35 of them are common with LSA. Applying thresholds on $V_{r}$ we get 761 empty polytopes and they contain all the failed epochs from the global test. Our method improves the reliability and the integrity of the navigation system at the cost of the continuity.

A comparison of MDBs obtained from both methods (Eq. 13 and 12.b) has been performed on the same test drive. The probability of error was $\alpha=0.002$ and the test quality (probability of type 2 error) was $\gamma=0.8$ which leads to a non-centrality parameter $\lambda_{i}=23,[1]$ and [25].

Figure 10, depicts the MDB for two satellites in view, a: PRN05 and b: PRN20. The $M D B_{P}$ of PRN05 is half of $M D B_{T S}$ (around $40 \%$ improvement) for most of the epochs and for some epochs where the geometry is quite good it has 65 $\%$ improvement. For PRN20 both methods show similar results for most of the epochs. However, there are many epochs when we have few satellites in view, so the $M D B_{T S}$ can not be computed while $M D B_{P}$ can be computed. For those epochs $M D B_{P}$ shows high values due to few constraints. As a results, $M D B_{P}$ improves the internal and the external reliability of the navigation system. 


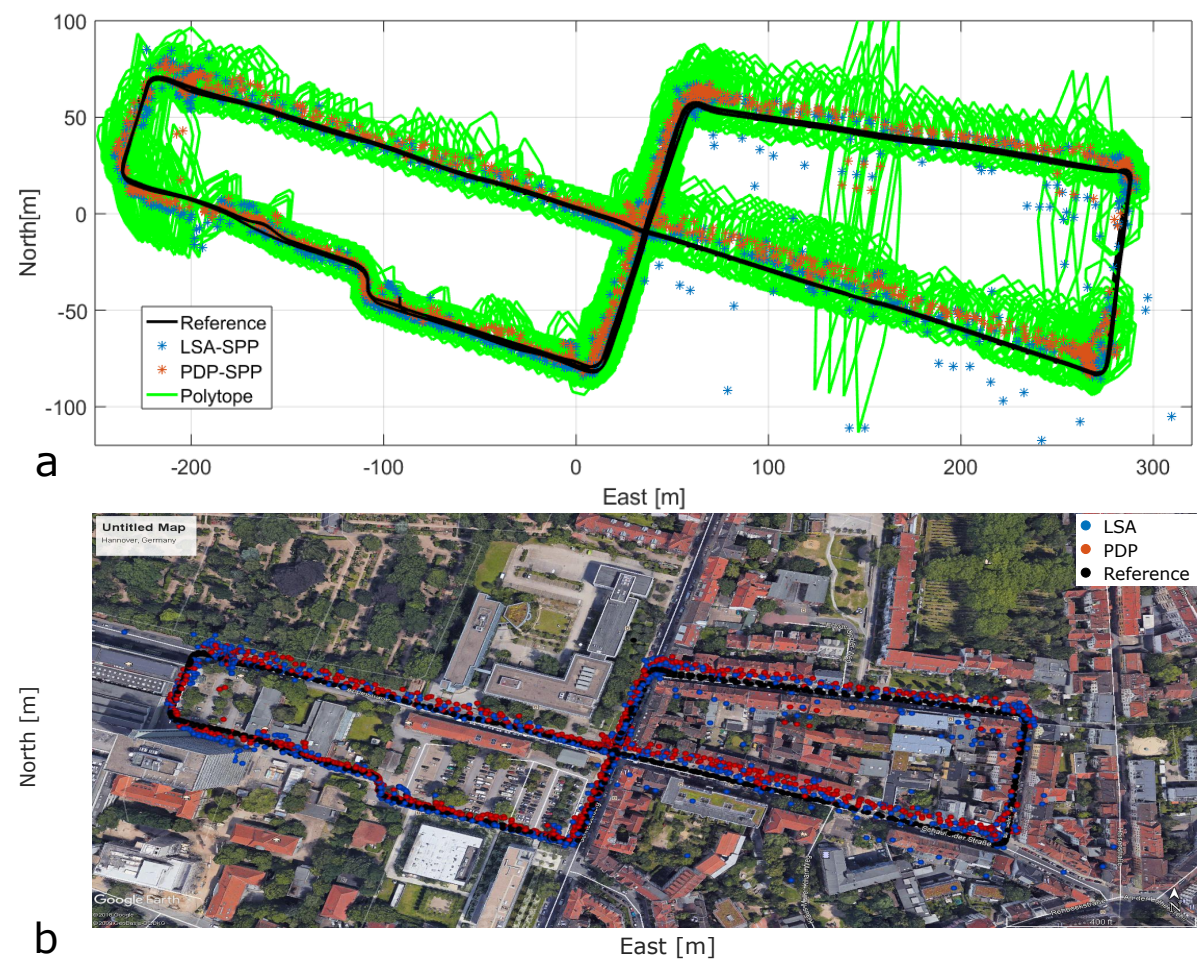

Figure 8: Results from test drives a: Positioning results and 2D projection of the obtained 4D polytopes for a test drive obtained with LSA and PDP algorithm. b: Google Earth of the test drive.

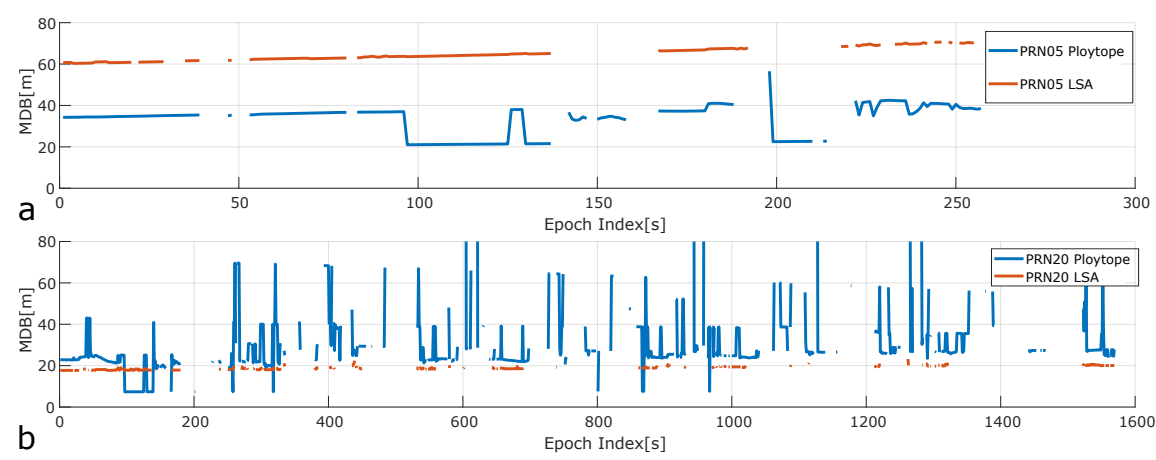

Figure 10: Minimum Detectable Bias computed from test statistic and from polytope for two satellites a) PRN05 and b) PRN20 


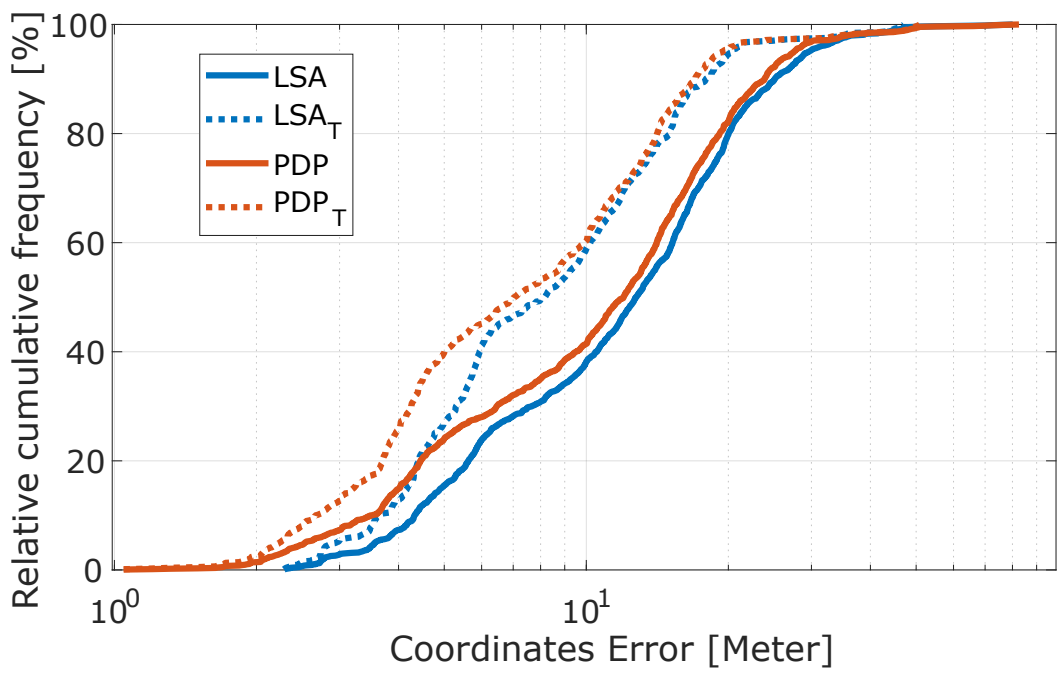

Figure 9: Cumulative frequency of the 3D coordinate errors, LSA (blue), PDP without applying a threshold on $V_{r}$ but excluding the epochs with empty sets (red) and PDP when a lower and an upper threshold are applied on $V_{r}\left(P D P_{T}\right.$ dashed red). For comparison, the statics of the LSA solution is shown when excluding all rejected epochs from $P D P_{T}\left(L S A_{T}\right.$, dashed blue).

\section{Conclusions}

In this paper, we presented steps towards an alternative approach for integrity monitoring. Associating deterministic error bands to the observations, we proposed a primal dual polytope algorithm to determine the feasible solution set. The resulting polytope represents the solution set. The volume of the polytope indicates the inconsistency of the observations, i.e. if the volume of the polytope is small, the observations are inconsistent. If the polytope is empty, large outliers are present. Exploiting the geometry of the navigation problem, zonotopes are derived as uncertainty reference and compared to the polytopes. Based on the comparison with a nominal error behavior (i.e. a zonotope), an outlier detection algorithm is developed and minimum detectable biases are indicated. Monte Carlo simulations indicate that the outlier detection performance depends on the ratio between the error bound and the standard deviation of the observations and on the geometry of the navigation problem.

Using real GPS code observations from a measurement campaign in an urban area with cars, we showed that the obtained polytopes always include the reference trajectory, while the classical least-squares solution shows large positioning deviations. In some of these cases, the polytopes were empty indicating the implicit outlier detection capability of the polytope approach. The internal reliability in terms of minimum detectable bias has been evaluated, too, applying the proposed 
new method and comparing it to the classical approach of global test statistics. The results show an reduction in MDB magnitude of up to $40 \%$, so smaller MDBs and thus higher sensitivity are obtained. Work on the inconsistency measures is in progress and fully integrity monitory will be developed in the near future.

\section{References}

[1] Baarda, W. Statistical concepts in geodesy, volume 2. Rijkscommissie voor Geodesie, 1967.

[2] Berg, D. Sten. https://www.terratec.se/, 2009.

[3] Binjammaz, T, Al-Bayatti, A, and Al-Hargan, A. GPS integrity monitoring for an intelligent transport system. In 10th Workshop on Positioning Navigation and Communication (WPNC). IEEE, 2013. DOI: 10.1109/WPNC. 2013.6533268.

[4] Boyd, S and Vandenberghe, L. Convex optimization. Cambridge university press, 2004. DOI: 10.1017/CB09780511804441.

[5] Cohen, J and Hickey, T. Two algorithms for determining volumes of convex polyhedra. Journal of the ACM (JACM), 26(3):401-414, 1979. DOI: 10.1145/322139.322141.

[6] Dbouk, H and Schön, S. Comparison of different bounding methods for providing GPS integrity information. In 2018 IEEE/ION Position, Location and Navigation Symposium (PLANS), pages 355-366. IEEE, 2018. DOI: 10.1109/PLANS . 2018.8373401.

[7] Dbouk, H and Schön, S. Reliability and integrity measures of GPS positioning via geometrical constraints. In Proceedings of the 2019 International Technical Meeting of The Institute of Navigation, pages 730-743, January 2019. DOI: 10.33012/2019.16722.

[8] Drevelle, V and Bonnifait, P. Reliable positioning domain computation for urban navigation. IEEE Intelligent Transportation Systems Magazine, 5(3):2129, 2013. DOI: $10.1109 /$ MITS.2013.2252058.

[9] Herceg, M, Kvasnica, M, Jones, C N, and Morari, M. Multi-parametric toolbox 3.0. In European Control Conference (ECC), 2013, pages 502-510. IEEE, 2013. DOI: $10.23919 /$ ECC.2013.6669862.

[10] iMAR IMU. https://www.novatel.com/assets/Documents/Papers/FSAS. pdf, 2016.

[11] Jaulin, L, Kieffer, M, Braems, I, and Walter, E. Guaranteed non-linear estimation using constraint propagation on sets. International Journal of Control, 74(18):1772-1782, 2001. DOI: 10.1080/00207170110090642. 
[12] Jaulin, L., Kieffer, M., Didrit, O., and Walter, E. Applied Interval Analysis. Springer-Verlag London, 2001. DOI: 10.1007/978-1-4471-0249-6.

[13] Kieffer, M, Jaulin, L, Walter, E, and Meizel, D. Robust autonomous robot localization using interval analysis. Reliable computing, 6(3):337-362, 2000. DOI: $10.1023 / \mathrm{A}: 1009990700281$.

[14] Klobuchar, J A. Ionospheric time-delay algorithm for single-frequency GPS users. Technical report, Air Force System Command Hanscom AFB MA Geophysics LAB, 1987. DOI: 10.1109/TAES.1987.310829.

[15] Langley, R. Dilution of precision. GPS world, 10(5):52-59, 1999.

[16] Lawrence, J. Polytope volume computation. Mathematics of Computation, 57(195):259-271, 1991. DOI: 10.1090/S0025-5718-1991-1079024-2.

[17] Parkinson, B W, Enge, P, Axelrad, P, and Spilker J, James J. Global positioning system: Theory and applications. American Institute of Aeronautics and Astronautics, 1996.

[18] Saastamoinen, J. Contributions to the theory of atmospheric refraction. Bulletin Géodésique (1946-1975), 107(1):13-34, $1973 . \quad$ DOI: 10.1007/BF02521844.

[19] Schön, S, Brenner, C, Alkhatib, H, Coenen, M, Dbouk, H, Garcia-Fernandez, N, Fischer, C, Heipke, C, Lohmann, K, Neumann, I, et al. Integrity and collaboration in dynamic sensor networks. Sensors, 18(7):2400, 2018. DOI: $10.3390 / \mathrm{s} 18072400$.

[20] Schön, S and Kutterer, H. Using zonotopes for overestimation-free interval least-squares - some geodetic applications. Reliable Computing, 11(2):137155, 2005. DOI: $10.1007 / \mathrm{s} 11155-005-3034-4$.

[21] Schön, S and Kutterer, H. Uncertainty in GPS networks due to remaining systematic errors: the interval approach. Journal of Geodesy, 80(3):150-162, 2006. DOI: $10.1007 / \mathrm{s} 00190-006-0042-z$.

[22] SPAN, NovAtel. https://www.novatel.com/assets/Documents/Manuals/ om-20000124.pdf, 2012.

[23] Speidel, J, Tossaint, M, Wallner, S, and Angel Avila-Rodriguz, J. Integrity for aviation: Comparing future concepts. Inside GNSS, 8(4):54-64, 2013.

[24] Teunissen, PJG. Minimal detectable biases of GPS data. Journal of Geodesy, 72(4):236-244, 1998. DOI: $10.1007 / \mathrm{s} 001900050163$.

[25] Teunissen, PJG. Testing theory: an introduction. Delft university press, VSSD, 2006. 
[26] Teunissen, PJG and Montenbruck, O, editors. Springer Handbook of Global Navigation Satellite Systems. Springer, Cham, 2017. DOI: 10.1007/978-3-319-42928-1.

[27] Wörner, M., Schuster, F., Dölitzscher, F., Keller, C., Haueis, M., and Dietmayer, K. Integrity for autonomous driving: A survey. In 2016 IEEE/ION Position, Location and Navigation Symposium (PLANS), pages 666-671, April 2016. DOI: 10.1109/PLANS . 2016.7479759.

[28] Ziegler, G.M. Lectures on Polytopes, volume 152. Springer, New York, NY, 1995. DOI: 10.1007/978-1-4613-8431-1. 